\title{
Investigation of the Relation Between Epithelial Barrier Function and Autism Symptom Severity in Children with Autism Spectrum Disorder
}

\section{Kevser Nalbant ( $\sim$ drkevser@yahoo.com )}

Hacettepe Universitesi Tip Fakultesi https://orcid.org/0000-0003-4261-1013

\section{Semih Erden}

Necmettin Erbakan Üniversitesi Meram Tıp Fakültesi: Necmettin Erbakan Universitesi Meram Tip Fakultesi

\section{Abdullah Yazar}

Necmettin Erbakan Üniversitesi Meram Tıp Fakültesi: Necmettin Erbakan Universitesi Meram Tip Fakultesi

İbrahim Kılınç

Necmettin Erbakan Üniversitesi Meram Tıp Fakültesi: Necmettin Erbakan Universitesi Meram Tip Fakultesi

\section{Research Article}

Keywords: Autism spectrum disorder, occludin, zonulin, epithelial barrier, gut-brain axis

Posted Date: November 30th, 2021

DOI: https://doi.org/10.21203/rs.3.rs-270289/v1

License: (9) This work is licensed under a Creative Commons Attribution 4.0 International License. Read Full License

Version of Record: A version of this preprint was published at Journal of Molecular Neuroscience on January 6th, 2022. See the published version at https://doi.org/10.1007/s12031-021-01954-z. 


\section{Abstract}

Autism spectrum disorder (ASD) is a common neurodevelopmental disorder characterized by limitations in mutual communication and social interaction as well as restricted, repetitive patterns of behaviors, interests, or activities. A number of biological abnormalities have been reported in ASD. Therefore the possible role of biological abnormalities in etiopathogenesis arouses research interest in this area. This is a case-control design study evaluating epithelial barrier function by comparing serum concentrations of occludin and zonulin in children with ASD $(n=60)$ and controls $(n=30)$. The Childhood Autism Rating Scale (CARS) was used to evaluate autistic symptom levels of all children. Serum occludin and zonulin levels were analyzed using an enzyme-linked immunosorbent assay. Serum occludin was significantly lower in children with ASD than in control subjects. In children with ASD, a decrease in occludin level was significantly associated with the disorder symptom levels item mean score (CARS total scores). Our findings showed that children with ASD had alterations in epithelial barrier function compared to the control group. The investigation of the mechanism underlying the different levels of occludin between ASD and controls may be of importance in clarifying the etiopathogenesis of ASD, as well as its follow-up and treatment.

\section{Introduction}

Autism spectrum disorder (ASD) is a neurodevelopmental disorder characterized by limitations in mutual communication and social interaction as well as restricted, repetitive patterns of behaviors, interests, or activities [1]. ASD prevalence has been reported to have increased and affecting approximately $2 \%$ of children [2]. Despite the increasing number of studies investigating this disorder, which negatively affects a significant part of the society [3], its etiopathogenesis remains uncertain and there is no satisfactory effective treatment. In addition, there is no biomarker for scanning, diagnosis, evaluation of the response to treatment, and/or follow-up of the disorder such as blood test, radiological scan. Therefore, studies to elucidate the biomarker of ASD have been increasing in recent years and many research areas have revealed abnormal biological processes associated with ASD [4]. Some previous studies have suggested that epithelial barrier dysfunction such as gut barrier may be a pathophysiological mechanism of ASD [59]

An imbalance in gut barrier integrity may trigger an immune reaction and may be associated with some diseases such as intestinal inflammation diseases, rheumatoid arthritis, and MS [10, 11]. Also, increasing numbers of studies indicate a possible link between ASD and disturbed gut barrier integrity [7-9]. In individuals with ASD, lactulose/mannitol ratio tests of gut permeability have shown increased gut permeability compared with healthy controls [7]. However, the mechanism of increased gut permeability in individuals with ASD has not been fully elucidated. Increasing evidence from many studies suggests that occludin $[12,13]$ and zonulin $[14,15]$ may be biomarkers of epithelial barrier permeability.

Occludin is an important tight junction (TJ) protein known to be associated with epithelial permeability [16]. Occludin contributes to the stability and integrity of TJs, thus regulating and restricting 
the paracellular transport pathway [17]. Occludin mutant or knockout animals exhibited chronic inflammation and a defective epithelial barrier despite having morphologically intact TJs; this demonstrated that it plays a critical role in maintaining barrier stability rather than TJ assembly [18, 19]. In a review of recent literature, there has been no study that evaluates serum occludin in ASD. When we looked at the studies on occludin, in a study that aimed to identify the biomarkers to track the active period in MS, serum levels of occludin and zonulin were found to increase during the active period of the disease, and it was emphasized that these findings supported the leaky gut hypothesis [16]. On the other hand, in inflammatory bowel disease studies, it has been reported that the expression of occludin decreases and this is related to gut permeability $[12,13]$.

Zonulin, a protein produced by small intestine epithelium, is known to induce TJ disassembly between enterocytes and then increase gut permeability [14]. Besides its effect on the gut, zonulin also regulates the blood-brain barrier (BBB) permeability[20] Therefore, it is being investigated as to whether it can be used as a biomarker of epithelial barrier permeability. Zonulin levels have been found to be high in obesity [21], Type 1 and 2 diabetes [22, 23], multiple sclerosis (MS) [16]. Also, zonulin has been studied in various psychiatric disorders such as schizophrenia [20,24], attention-deficit hyperactivity disorder [25, $26]$, obsessive-compulsive disorders [27], and ASD [28,29]. The results of previous clinical studies on zonulin levels in psychiatric disorders are inconsistent. Some studies have found that patients have higher levels of zonulin compared to controls [28, 30, 25, 20]; other studies have found no difference between the two groups $[29,27]$.

These contradictory results suggest that more research is needed on occludin and zonulin. Therefore, based on previous studies suggesting that the epithelial barrier permeability of occludin and zonulin may be biomarkers, we aimed to evaluate epithelial barrier function by comparing the serum concentrations of occludin and zonulin in children with ASD and control groups. To the best of our knowledge, there has been no study in the current literature that evaluates serum occludin and zonulin levels together in ASD. We assumed that increased levels of occludin and zonulin could happen in ASD, and the severity of the ASD could be related to occludin and zonulin levels. Since zonulin is a regulator of the TJ proteins, we assumed that the levels of occludin and zonulin would correlate positively with each other. Then, we discussed further the possible causes of changes in the levels of these biomarkers.

\section{Materials And Methods}

\section{Participants}

This study was conducted at the ...'our institution', Department of Child and Adolescent Psychiatry and Department of Pediatrics between March and December 2018. The diagnosis of ASD was made through clinical psychiatric evaluations by a child and adolescent psychiatrist based on the the semi-structured psychiatric interview form of the Schedule for Affective Disorders and Schizophrenia for School-Age Children-Present and Lifetime Version (K-SADS-PL) / DSM-5 5th Edition (DSM-5). Of the children aged between 30-96 months who were diagnosed as having ASD, only those whose parents had agreed to 
participate in the study were included. Children newly diagnosed as having ASD and children with known ASD under follow up were divided into two groups. In order to control for confounding variables, children who took medicine, those diagnosed as having a GI disease (e.g. Crohn's disease, ulcerative colitis), chronic disease (e.g. diabetes mellitus, hypertension, epilepsy, cerebral palsy), and children with infections or obesity were excluded from the study. All parents in the study volunteered to participate at no cost. All children with ASD who met the inclusion criteria were invited to join the study. There were no parents who refused to participate in the study. A total of 90 children, consisting of 60 children with ASD and 30 controls, were included in this study. Among 72 with the provisional diagnosis of ASD, six children with ASD were excluded because of the diagnosis of epilepsy, four children with ASD due to an accompanying severe medical disease, and two children with ASD did not meet the DSM-5 diagnostic criteria of ASD.

For the control group, children admitted to ...'our institution', Department of Pediatrics outpatient clinics in aged between 30-96 months, whose laboratory tests (hemogram, sedimentation, C-reactive protein) and examinations were normal, who were not on medication, who had no chronic disease (e.g. diabetes mellitus, hypertension, epilepsy, cerebral palsy), no infectious disease, GI disease or obesity were referred to ..'our institution' Department of Child and Adolescent Psychiatry. Children without any psychiatric disease, according to the semi-structured psychiatric interview form of the K-SADS-PL / DSM-5 were included in the study. Written consents were obtained from the parents of all children included in the study. The Sociodemographic Data Form and the Turkish version of the Childhood Autism Rating Scale (CARS) were completed during the clinical psychiatric evaluation by the child and adolescent psychiatrist. Among 46 children who parents volunteered to participate in the control group, sixteen were excluded because of a psychiatric disorder diagnosed through a semi-structured clinical interview (K-SADS-PL / DSM-5). The evaluation of each participant took about 2 hours.

\section{Instruments}

\section{Sociodemographic and clinical information form}

The sociodemographic form included age, sex, birth history, developmental history, medical history, family history, educational status, and medications used.

\section{Childhood Autism Rating Scale (CARS)}

CARS, a semi-structured assessment tool, composed of 15 items. It is rated by physicians according to the information obtained from child observations and family interviews [31]. A reliability and validity study of CARS for the our population was conducted by' ...'

\section{Schedule for Affective Disorders and Schizophrenia for School-Age Children-Present and Lifetime Version (K-SADS-PL / DSM-5).}

The K-SADS-PL is a semi-structured clinical interview used for determining the present and lifetime psychopathologies of children and adolescent (Kaufman et al., 2016). A reliability and validity study of K- 
SADS-PL for the our population was conducted by ' ...'

\section{Serum samples}

Serum samples were obtained between 08:30 and 11:00 AM. Samples were stored at $-80^{\circ} \mathrm{C}$ until the study. Serum occludin and zonulin levels were measured using the sandwich-enzyme-linked immunosorbent assay method (Human Zonulin ELISA Kit, Catalogue No: E-EL-H5560, USA) (Human occludin ELISA Kit, Catalogue No: E-EL-H1073, USA). Results are given in ng/mL.

\section{Statistical Analysis}

All statistical analyses were performed using the Statistical Package for the Social Sciences (SPSS) 15.0 for Windows software (SPSS Inc., Chicago, Illinois). The normality of the data was assessed using the Kolmogorov-Smirnov test and Skewness and Kurtosis statistics. For parametric data in the evaluation of the data; Student's t-test, ANOVA (for variance analysis), Pearson's correlation test (for the relationship of data) were applied. For nonparametric data; Mann Whitney U test and Spearman's correlation test (for the relationship of data) were used. The Chi-square $\left(\mathrm{c}^{2}\right)$ test was used for categorical variable. $P$ values were expected to be less than 0.05 for statistical significance in all analyses.

\section{Results}

A total of 90 children aged between 30-96 months, consisting of 60 children with ASD and 30 controls, were included in this study. There were no significant differences between the two groups in terms of age, sex, and socioeconomic level. Sociodemographic data and clinical characteristics are presented in Table 1. Serum occludin levels were found to be statistically significantly lower in children with ASD $(0.83 \pm$ $0.94)(p<0.001)$. There was no statistically significant difference in serum zonulin levels (Table 1$)$.

The serum occludin and zonulin levels of the children with newly diagnosed ASD and the children followed up with ASD were compared with the control group. Accordingly, serum occludin levels were statistically lower both in children with new diagnoses $(0.75 \pm 0.81)(p<0.001)$ and children with previously diagnosed ASD $(0.80 \pm 0.96)(p<0.001)$. There was no statistically significant difference between the three groups in terms of serum zonulin levels (Table 2). Furthermore, it was investigated whether there was a difference in terms of occludin and zonulin levels between males (Table 3 ) and females (Table 4) with ASD group and the control group. Statistical analyzes were found to be similar to analyzes with all children with ASD group and the control group.

The correlation between serum occludin and zonulin levels and the CARS total scores of the children in all groups was evaluated. There was no statistically significant correlation between serum zonulin and occludin levels. A statistically significant negative and moderate correlation was found between serum occludin levels and CARS scores $(p<0.001)$. There was no statistically significant correlation between serum zonulin levels and CARS scores (Table 5). 
The correlation between serum occludin and zonulin levels and the CARS total scores of the children with ASD group was also evaluated. There was no statistically significant correlation between serum zonulin and occludin levels $(r=0.063, p>0.05)$. There was no statistically significant correlation between serum occludin and zonulin levels and CARS total score $(r=0.033, r=-0.161$ respectively).

\section{Discussion}

In this study, serum levels of occludin, which is a paracellular TJ protein, and zonulin, which functions as the regulator of the paracellular TJ proteins, were compared between children with ASD and control children of similar age, sex, and socioeconomic level. According to our findings, the serum occludin levels in children with ASD were lower than in the control group, but there was no significant difference between the groups in terms of serum zonulin levels. When the relationship of both markers to CARS scores was evaluated, it was found that the CARS scores increased as the occludin levels decreased, but no significant relationship was found between zonulin and CARS scores.

Occludin, is part of the epithelial and endothelial junction complexes, and recent data suggest that occludin regulates barrier functions of structures such as the gut and BBB [32]. In a review of recent literature, no study investigating the relationship between occludin and ASD was found. When we looked at the studies on occludin, in a study that aimed to identify the biomarkers to watch the active period in MS, serum levels of occludin and zonulin were found to increase during the active period of the disease, and it was emphasized that these findings supported the leaky gut hypothesis [16]. A study by Mankertz et al. showed that proinflammatory cytokines [tumor necrosis factor-alpha (TNF-a), interferon gamma (IFN- $\mathrm{\gamma})$ ] down regulated occludin expression, and the authors suggested that increased proinflammatory cytokines in inflammatory diseases might be an important mechanism in the increased paracellular TJ permeability by mediating the reduction of occludin expression [33]. In two other studies, in which gut barrier function was investigated in inflammatory diseases of the intestines (Crohn's and ulcerative colitis), it was stated that occludin expression decreased during the active period of the disease, unlike other intracellular TJ proteins, and this might play a role in increased paracellular permeability [12, 13]. In addition to these studies, in vivo and in vitro studies have shown that proinflammatory cytokines also disrupt TJs in the BBB $[34,35]$. The results of all these studies appear to correlate with the fact that inflammatory processes disrupt TJ proteins, specifically reducing occludin expression. Moreover, in recent years, emphasis has been increasing on the role of inflammatory processes in ASD etiopathogenesis [36]. In fact, a recent meta-analysis concluded that the concentration of pro-inflammatory cytokines [IFN- $\gamma$, interleukin-1 (IL-1), IL-6, and TNF-a] in individuals diagnosed with ASD was higher than that of controls [37]. Given these findings, although simultaneous proinflammatory cytokines were not examined in our study, it may be suggested that the low levels of occludin in children with ASD in our study may be the result of increased inflammation. However, this assumption needs to be supported by looking at the simultaneous levels of proinflammatory cytokine and occludin. In addition, the results of our study should be investigated in more detail with gut biopsy samples, in vivo and in vitro studies in a larger sample. 
Another biomarker, zonulin has been suggested for use as a serologic marker for the evaluation of epithelial barrier integrity. Zonulin is produced by enterocytes under the influence of environmental stimuli [14], and mediates increased intestinal permeability through TJs $[14,15]$. The results of previous clinical studies on zonulin levels in ASD are inconsistent. There are studies reporting that serum zonulin levels are higher in children with ASD compared with typically developing children [28, 30], as well as studies that reported that they were not different [29]. In a recent study, in vitro IFN-, IL-17A or zonulin exposure was found to increase the small intestine epithelial barrier and BBB permeability by changing the localization of zonula occludens, claudine-5, and occludin, and it was stated that these findings might help to explain the pathogenesis of neuro-inflammatory diseases associated with disorders in the gutbrain axis [34]. Given the studies reporting that intestinal permeability plays a role in ASD, it may be considered that zonulin could contribute to the development of ASD through this mechanism. However, there was no statistically significant difference between the groups in terms of zonulin levels. This findings suggest that the rates of construction and destruction of zonulin were similar to that of between the ASD and control group.

Finally, since zonulin is a regulator of the TJ proteins, we assumed that the levels of occludin and zonulin would correlate positively with each other. However, no correlation was found between occludin and zonulin levels in our study. Based on our findings, we can infer that the difference in occludin levels was independent of zonulin. We believe that occludin's involvement in both the gut barrier and the BBB may mediate our understanding of the pathogenesis of ASD associated with the gut-brain axis. In addition, clarification of the structure represented by occludin subtypes through genetic research will facilitate the interpretation of these results.

Despite the inclusion of more participants with ASD than in previous studies, our study is still limited. The main limitation of the study is it's cross-sectional rather than longitudinal design. This does not allow us to find any cause-effect for our results. The absence of an examination of simultaneous proinflammatory cytokines, the absence of objective tests of intestinal permeability (lactulose/mannitol ratio tests), and the absence of an examination of intestinal biopsy and epithelial barriers were among the other limitations of our study. Further studies with larger sample sizes are required to reach stronger conclusions. Despite all these limitations, our findings suggest that occludin may play a role in influencing the gut-brain axis in children with ASD.

The serum levels of occludin and zonulin were detected in this study. Our findings have shown that there are alterations of the occludin levels in children with ASD. Further studies are needed to investigate the role of occludin in influencing the gut-brain axis in children with ASD. Previous studies suggest that biological abnormalities may be associated with ASD and these abnormalities may be important in diagnosis, follow-up, and/or treatment as potential biomarkers. The investigation of the mechanism underlying the different levels of occludin between ASD and controls may be of importance in clarifying the etiopathogenesis of ASD, as well as its follow-up and treatment.

\section{Declarations}


Acknowledgements

We wish to thank all subjects who participated in this study.

\section{Funding details}

Funding for this study was provided by a grant from the Scientific Research Project Coordination Unit of ...'our institution' (Project no: 181218011)

\section{Conflict of interest}

On behalf of all authors, the corresponding author states that there is no conflict of interest.

\section{Availability of data}

The data used to support the findings of this study are available from the corresponding author upon request.

\section{Authors' contributions}

All authors have made a significant contribution to the findings, writing, and methods in the paper.

\section{Ethical approval}

All procedures performed in studies involving human participants were in accordance with the ethical standards of the institutional and/or national research committee and with the 1964 Helsinki Declaration and its later amendments or comparable ethical standards. This research was approved by the Ethics Committee of the Non-Interventional Clinical Research of ...'our institution'

\section{Informed consent}

Informed consent was obtained from all individual participants included in the study.

\section{References}

1. Association AP. Diagnostic and statistical manual of mental disorders (DSM-5®). American Psychiatric Pub; 2013.

2. Xu G, Strathearn L, Liu B, O'Brien M, Kopelman TG, Zhu J et al. Prevalence and Treatment Patterns of Autism Spectrum Disorder in the United States, 2016. JAMA Pediatr. 2019;173(2):153-9. doi:10.1001/jamapediatrics.2018.4208.

3. Rogge N, Janssen J. The Economic Costs of Autism Spectrum Disorder: A Literature Review. J Autism Dev Disord. 2019;49(7):2873-900. doi:10.1007/s10803-019-04014-z.

4. Frye RE, Vassall S, Kaur G, Lewis C, Karim M, Rossignol D. Emerging biomarkers in autism spectrum disorder: a systematic review. Ann Transl Med. 2019;7(23):792. doi:10.21037/atm.2019.11.53. 
5. Erickson CA, Stigler KA, Corkins MR, Posey DJ, Fitzgerald JF, McDougle CJ. Gastrointestinal factors in autistic disorder: a critical review. J Autism Dev Disord. 2005;35(6):713-27. doi:10.1007/s10803005-0019-4.

6. Liu Z, Li N, Neu J. Tight junctions, leaky intestines, and pediatric diseases. Acta Paediatr. 2005;94(4):386-93. doi:10.1111/j.1651-2227.2005.tb01904.x.

7. de Magistris L, Familiari V, Pascotto A, Sapone A, Frolli A, lardino P et al. Alterations of the intestinal barrier in patients with autism spectrum disorders and in their first-degree relatives. J Pediatr Gastroenterol Nutr. 2010;51(4):418-24. doi:10.1097/MPG.0b013e3181dcc4a5.

8. Iovene MR, Bombace F, Maresca R, Sapone A, lardino P, Picardi A et al. Intestinal Dysbiosis and Yeast Isolation in Stool of Subjects with Autism Spectrum Disorders. Mycopathologia. 2017;182(3-4):34963. doi:10.1007/s11046-016-0068-6.

9. Fiorentino M, Sapone A, Senger S, Camhi SS, Kadzielski SM, Buie TM et al. Blood-brain barrier and intestinal epithelial barrier alterations in autism spectrum disorders. Mol Autism. 2016;7:49. doi:10.1186/s13229-016-0110-z.

10. De Mey JR, Freund JN. Understanding epithelial homeostasis in the intestine: An old battlefield of ideas, recent breakthroughs and remaining controversies. Tissue Barriers. 2013;1(2):e24965. doi:10.4161/tisb.24965.

11. Catalioto RM, Maggi CA, Giuliani S. Intestinal epithelial barrier dysfunction in disease and possible therapeutical interventions. Curr Med Chem. 2011;18(3):398-426. doi:10.2174/092986711794839179.

12. Kucharzik T, Walsh SV, Chen J, Parkos CA, Nusrat A. Neutrophil transmigration in inflammatory bowel disease is associated with differential expression of epithelial intercellular junction proteins. Am J Pathol. 2001;159(6):2001-9. doi:10.1016/S0002-9440(10)63051-9.

13. Zeissig S, Burgel N, Gunzel D, Richter J, Mankertz J, Wahnschaffe U et al. Changes in expression and distribution of claudin 2, 5 and 8 lead to discontinuous tight junctions and barrier dysfunction in active Crohn's disease. Gut. 2007;56(1):61-72. doi:10.1136/gut.2006.094375.

14. Fasano A. Zonulin and its regulation of intestinal barrier function: the biological door to inflammation, autoimmunity, and cancer. Physiol Rev. 2011;91(1):151-75. doi:10.1152/physrev.00003.2008.

15. Tripathi A, Lammers KM, Goldblum S, Shea-Donohue T, Netzel-Arnett S, Buzza MS et al. Identification of human zonulin, a physiological modulator of tight junctions, as prehaptoglobin-2. Proc Natl Acad Sci U S A. 2009;106(39):16799-804. doi:10.1073/pnas.0906773106.

16. Camara-Lemarroy CR, Silva C, Greenfield J, Liu WQ, Metz LM, Yong VW. Biomarkers of intestinal barrier function in multiple sclerosis are associated with disease activity. Mult Scler. 2019:1352458519863133. doi:10.1177/1352458519863133.

17. Feldman GJ, Mullin JM, Ryan MP. Occludin: structure, function and regulation. Adv Drug Deliv Rev. 2005;57(6):883-917. doi:10.1016/j.addr.2005.01.009. 
18. Balda MS, Flores-Maldonado C, Cereijido M, Matter K. Multiple domains of occludin are involved in the regulation of paracellular permeability. J Cell Biochem. 2000;78(1):85-96.

19. Chen Y, Merzdorf C, Paul DL, Goodenough DA. $\mathrm{COOH}$ terminus of occludin is required for tight junction barrier function in early Xenopus embryos. J Cell Biol. 1997;138(4):891-9. doi:10.1083/jcb.138.4.891.

20. Barber GS, Sturgeon C, Fasano A, Cascella NG, Eaton WW, McMahon RP et al. Elevated zonulin, a measure of tight-junction permeability, may be implicated in schizophrenia. Schizophr Res. 2019;211:111-2. doi:10.1016/j.schres.2019.07.006.

21. Zak-Golab A, Kocelak P, Aptekorz M, Zientara M, Juszczyk L, Martirosian G et al. Gut microbiota, microinflammation, metabolic profile, and zonulin concentration in obese and normal weight subjects. Int J Endocrinol. 2013;2013:674106. doi:10.1155/2013/674106.

22. Jayashree B, Bibin YS, Prabhu D, Shanthirani CS, Gokulakrishnan K, Lakshmi BS et al. Increased circulatory levels of lipopolysaccharide (LPS) and zonulin signify novel biomarkers of proinflammation in patients with type 2 diabetes. Mol Cell Biochem. 2014;388(1-2):203-10. doi:10.1007/s11010-013-1911-4.

23. Sapone A, de Magistris L, Pietzak M, Clemente MG, Tripathi A, Cucca F et al. Zonulin upregulation is associated with increased gut permeability in subjects with type 1 diabetes and their relatives. Diabetes. 2006;55(5):1443-9. doi:10.2337/db05-1593.

24. Usta A, Kilic F, Demirdas A, Isik U, Doguc DK, Bozkurt M. Serum zonulin and claudin-5 levels in patients with schizophrenia. Eur Arch Psychiatry Clin Neurosci. 2021;271(4):767-73. doi:10.1007/s00406-020-01152-9.

25. Ozyurt G, Ozturk Y, Appak YC, Arslan FD, Baran M, Karakoyun I et al. Increased zonulin is associated with hyperactivity and social dysfunctions in children with attention deficit hyperactivity disorder. Compr Psychiatry. 2018;87:138-42. doi:10.1016/j.comppsych.2018.10.006.

26. Aydogan Avsar P, Isik U, Aktepe E, Kilic F, Doguc DK, Buyukbayram HI. Serum zonulin and claudin-5 levels in children with attention-deficit/hyperactivity disorder. Int J Psychiatry Clin Pract. 2021;25(1):49-55. doi:10.1080/13651501.2020.1801754.

27. Isik U, Aydogan Avsar P, Aktepe E, Doguc DK, Kilic F, Buyukbayram HI. Serum zonulin and claudin-5 levels in children with obsessive-compulsive disorder. Nord J Psychiatry. 2020:1-6. doi:10.1080/08039488.2020.1715474.

28. Esnafoglu E, Cirrik S, Ayyildiz SN, Erdil A, Erturk EY, Dagli A et al. Increased Serum Zonulin Levels as an Intestinal Permeability Marker in Autistic Subjects. J Pediatr. 2017;188:240-4. doi:10.1016/j.jpeds.2017.04.004.

29. Jozefczuk J, Konopka E, Bierla JB, Trojanowska I, Sowinska A, Czarnecki R et al. The Occurrence of Antibodies Against Gluten in Children with Autism Spectrum Disorders Does Not Correlate with Serological Markers of Impaired Intestinal Permeability. J Med Food. 2018;21(2):181-7. doi:10.1089/jmf.2017.0069. 
30. Rose DR, Yang H, Serena G, Sturgeon C, Ma B, Careaga M et al. Differential immune responses and microbiota profiles in children with autism spectrum disorders and co-morbid gastrointestinal symptoms. Brain Behav Immun. 2018;70:354-68. doi:10.1016/j.bbi.2018.03.025.

31. Schopler E, Reichler RJ, DeVellis RF, Daly K. Toward objective classification of childhood autism: Childhood Autism Rating Scale (CARS). Journal of autism and developmental disorders. 1980.

32. Raleigh DR, Boe DM, Yu D, Weber CR, Marchiando AM, Bradford EM et al. Occludin S408 phosphorylation regulates tight junction protein interactions and barrier function. J Cell Biol. 2011;193(3):565-82. doi:10.1083/jcb.201010065.

33. Mankertz J, Tavalali S, Schmitz H, Mankertz A, Riecken EO, Fromm M et al. Expression from the human occludin promoter is affected by tumor necrosis factor alpha and interferon gamma. $\mathrm{J}$ Cell Sci. 2000;113 ( Pt 11):2085-90.

34. Rahman MT, Ghosh C, Hossain M, Linfield D, Rezaee F, Janigro D et al. IFN-gamma, IL-17A, or zonulin rapidly increase the permeability of the blood-brain and small intestinal epithelial barriers: Relevance for neuro-inflammatory diseases. Biochem Biophys Res Commun. 2018;507(1-4):274-9. doi:10.1016/j.bbrc.2018.11.021.

35. Kebir H, Kreymborg K, Ifergan I, Dodelet-Devillers A, Cayrol R, Bernard M et al. Human TH17 lymphocytes promote blood-brain barrier disruption and central nervous system inflammation. Nat Med. 2007;13(10):1173-5. doi:10.1038/nm1651.

36. Shen Y, Li Y, Shi L, Liu M, Wu R, Xia K et al. Autism spectrum disorder and severe social impairment associated with elevated plasma interleukin-8. Pediatr Res. 2020. doi:10.1038/s41390-020-0910-x.

37. Saghazadeh A, Ataeinia B, Keynejad K, Abdolalizadeh A, Hirbod-Mobarakeh A, Rezaei N. A metaanalysis of pro-inflammatory cytokines in autism spectrum disorders: Effects of age, gender, and latitude. J Psychiatr Res. 2019;115:90-102. doi:10.1016/j.jpsychires.2019.05.019.

\section{Tables}

TABLE 1. Sociodemographic and clinical characteristics of participants

TABLE 2. Occludin and zonulin levels of newly diagnosed with ASD, followed up with ASD and controls

\begin{tabular}{|c|c|c|c|c|c|}
\hline & $\begin{array}{l}\text { Newly diagnosed with } \\
\text { ASD }(n=30)\end{array}$ & $\begin{array}{l}\text { Followed up with } \\
\text { ASD }(n=30)\end{array}$ & $\begin{array}{l}\text { Control } \\
(n=30)\end{array}$ & Statistics & $\mathrm{p}$ \\
\hline $\begin{array}{l}\text { Occludin } \\
(\mathrm{ng} / \mathrm{mL}) \text {, Mean } \\
( \pm S D)\end{array}$ & $0.75 \pm 0.81$ & $0.80 \pm 0.96$ & $\begin{array}{l}2.30 \pm \\
1.48\end{array}$ & $\begin{array}{l}F= \\
26.810\end{array}$ & 0.00 \\
\hline $\begin{array}{l}\text { Zonulin } \\
(\mathrm{ng} / \mathrm{mL}) \text {, Mean } \\
( \pm S D)\end{array}$ & $10.71 \pm 16.06$ & $8.46 \pm 12.13$ & $\begin{array}{l}14.91 \pm \\
25.03\end{array}$ & $F=0.333$ & 0.33 \\
\hline
\end{tabular}




\begin{tabular}{|c|c|c|c|c|}
\hline & $\begin{array}{c}\text { ASD } \\
\text { N/Mean } \\
(\% / \pm S D)\end{array}$ & $\begin{array}{l}\text { Control } \\
\text { N/Mean } \\
(\% / \pm S D)\end{array}$ & Statistics & $\mathrm{p}$ \\
\hline Age (months) & $\begin{array}{l}53.40 \pm \\
22.65\end{array}$ & $19.69^{60.60 \pm}$ & $-1.482^{t=}$ & 0.142 \\
\hline \multicolumn{5}{|l|}{ Sex } \\
\hline Male & $51(85)$ & $21(70)$ & \multirow{2}{*}{$2.813^{x^{2}=}$} & \multirow[t]{2}{*}{0.094} \\
\hline Female & $9(15)$ & $9(30)$ & & \\
\hline Mother's age & $\begin{array}{l}32.58 \\
(5.39)\end{array}$ & $\begin{array}{l}32.70 \\
(5.22)\end{array}$ & $0.265^{t=}$ & 0.92 \\
\hline Mother's education, years & $(4.02)^{8.51}$ & $9.20(4.36)$ & $t=-0.94$ & 0.56 \\
\hline Father's age & $\begin{array}{l}35.24 \\
(5.39)\end{array}$ & $(5.62)^{37.16}$ & $-1.435^{t=}$ & 0.15 \\
\hline Father's education, years & $\begin{array}{l}10.02 \\
(4.46)\end{array}$ & $9.45(3.92)$ & $2.731^{t=}$ & 0.63 \\
\hline $\begin{array}{l}\text { Age of starting to } \\
\text { walk (months) }\end{array}$ & $15.4 \pm 5.8$ & $14.5 \pm 4.2$ & $t=0.97$ & 0.23 \\
\hline $\begin{array}{l}\text { Age of starting to } \\
\text { speak, (months) }\end{array}$ & $10.9^{22.3 \pm}$ & $25.3 \pm 11.3$ & $t=-1.20$ & 0.78 \\
\hline Occludin, (ng/mL) & $\begin{array}{l}0.83 \\
(0.94)^{0.0}\end{array}$ & $2.30(1.48)$ & $\begin{array}{r}U= \\
267.000\end{array}$ & 0.000 \\
\hline Zonulin (ng/mL) & $\begin{array}{l}8.96 \\
(13.65)\end{array}$ & $\begin{array}{l}14.91 \\
(25.03)\end{array}$ & $\begin{array}{r}U= \\
719.500\end{array}$ & 0.24 \\
\hline
\end{tabular}

ASD: Autistic spectrum disorder, SD: Standard deviation 
TABLE 3: Occludin and Zonulin levels of males with ASD and controls

\begin{tabular}{|lllll|}
\hline & ASD & Control & Statistics & $p$ \\
& $(\mathrm{n}=51)$ & $(\mathrm{n}=21)$ & & \\
\hline Occludin $(\mathrm{ng} / \mathrm{mL})$, Mean $( \pm \mathrm{SD})$ & $0.82 \pm 0.97$ & $2.24 \pm 1.40$ & $\mathrm{U}=169,500$ & $\mathbf{0 . 0 0 0}$ \\
& & & & \\
& & & & \\
\hline Zonulin $(\mathrm{ng} / \mathrm{mL})$, Mean $( \pm S D)$ & $9.82 \pm 14.59$ & $15.74 \pm 27.91$ & $\mathrm{U}=488,000$ & 0.55 \\
\hline
\end{tabular}

ASD: Autistic spectrum disorder, SD: Standard deviation

TABLE 4: Occludin and zonulin levels of females with ASD and controls

\begin{tabular}{|lllll|}
\hline & ASD & Control & Statistics & $p$ \\
& $(\mathrm{n}=9)$ & $(\mathrm{n}=9)$ & & \\
\hline Occludin $(\mathrm{ng} / \mathrm{mL})$, Mean $( \pm S D)$ & $0.84 \pm 0.82$ & $2.21 \pm 1.67$ & $\mathrm{U}=18.000$ & $\mathbf{0 . 0 4 7}$ \\
& & & & \\
& & & & \\
\hline Zonulin $(\mathrm{ng} / \mathrm{mL})$, Mean $( \pm S D)$ & $4.09 \pm 3.67$ & $10.01 \pm 13.49$ & $\mathrm{U}=30.000$ & 0.38 \\
\hline
\end{tabular}

ASD: Autistic spectrum disorder, SD: Standard deviation

TABLE 5. Correlations of CARS total score with serum Occludin and zonulin levels among all participants

\begin{tabular}{|llll|}
\hline & Occludin & Zonulin & CARS \\
\hline Occludin & 1 & & \\
\hline Zonulin & .123 & 1 & \\
\hline CARS & $-.436 * *$ & -.112 & 1 \\
\hline
\end{tabular}

CARS: Childhood Autism Rating Scale, ** $p<0.001$ 